\title{
Topological flat bands and correlated states in twisted monolayer-bilayer graphene
}

\author{
Louk Rademaker, Ivan V. Protopopov, and Dmitry A. Abanin \\ Department of Theoretical Physics, University of Geneva, 1211 Geneva, Switzerland
}

(Received 6 May 2020; revised 29 June 2020; accepted 30 June 2020; published 27 July 2020)

\begin{abstract}
Monolayer graphene placed with a twist on top of AB-stacked bilayer graphene hosts topological flat bands in a wide range of twist angles. The dispersion of these bands and gaps between them can be efficiently controlled by a perpendicular electric field, which induces topological transitions accompanied by changes of the Chern numbers. In the regime where the applied electric field induces gaps between the flat bands, we find a relatively uniform distribution of the Berry curvature. Consequently, interaction-induced valley- and/or spin-polarized states at integer filling factors are energetically favorable. In particular, we predict a quantum anomalous Hall state at filling factors $v=1,3$ for a range of twist angles $1^{\circ}<\theta<1.4^{\circ}$. Furthermore, to characterize the response of the system to magnetic field, we computed the Hofstadter butterfly and the Wannier plot, which can be used to probe the dispersion and topology of the flat bands in this material.
\end{abstract}

DOI: 10.1103/PhysRevResearch.2.033150

\section{INTRODUCTION}

Studies of twisted two-dimensional materials have gained an enormous boost after the discovery of correlated insulator states and superconductivity in twisted bilayer graphene (tBG) [1-6]. tBG has been predicted [7] to be a natural platform for interaction physics due to the presence of magic angles where nearly flat bands near charge neutrality are formed. Following these results, a variety of related stacked materials with flat bands have been proposed and experimentally studied, including twisted double graphene bilayers [8], trilayer graphene on hBN [9-11], and twisted transition-metal dichalcogenides [12].

Several twisted materials naturally lead to topologically nontrivial band structures [13] and may provide platforms for the quantum anomalous Hall (QAH) effect [14]. Standard $\mathrm{tBG}$ is one of the first materials that display "fragile topology" [15-18]. Trilayer graphene on hBN can exhibit nontrivial Chern bands under an applied field [9-11]. Further, recent experiments observed striking signatures of the QAH effect in tBG $[19,20]$. Theoretical Hartree-Fock studies [21-23] complemented with exact diagonalization studies [24] have argued that such a state may arise in $\mathrm{tBG}$ as a combined effect of alignment with the hBN substrate and Coulomb interaction, via a mechanism reminiscent of quantum Hall ferromagnetism.

A major challenge, however, is that the appearance of Chern bands appears to be very sensitive to model details. For example, Hartree-Fock band renormalization effects changes the Chern number of bands in trilayer graphene [9]. The circumstances under which the $\mathrm{hBN}$ substrate gives rise to

Published by the American Physical Society under the terms of the Creative Commons Attribution 4.0 International license. Further distribution of this work must maintain attribution to the author(s) and the published article's title, journal citation, and DOI.
Chern bands in tBG are still not fully understood [21,23]. With this in mind, in this article we carry out a detailed study of a twisted candidate material [25-27]: monolayer graphene twisted with respect to AB-stacked bilayer graphene, called twisted monolayer-bilayer graphene (tMBG).

We find that for realistic hopping parameters and a wide range of twist angles, this system hosts isolated flat bands separated by energy gaps from the remaining bands. In each valley, the two flat bands have a net nonzero Chern number, in accordance with predictions of Ref. [25] for twisted $N+M$ layers of graphene. The flat bands can be efficiently controlled by gates: A perpendicular electric field allows us to tune both the dispersion of the flat bands and gaps between them. Even more interestingly, realistic electric fields can induce a series of topological transitions, accompanied by changes of Chern numbers of the flat bands, and redistribution of the Berry's curvature in the Brillouin zone. The topological character of the flat bands gives rise to a characteristic response to a perpendicular magnetic field, having clear signatures in the Hofstadter butterfly, which we compute.

Finally, we show using Hartree-Fock calculations that interactions will favor spin- and/or valley-polarized states in a broad regime of twist angles. In particular, states with a filling factor $v=1$ are found to be both spin and valley polarized, exhibiting a QAH effect. Their stability in a broad range of angles and tunability by gates make tMBG a promising candidate for realizing robust QAH effect in a sample-independent manner. We note that previous works [28-30] investigated electronic properties of trilayer graphene where both top and bottom layers are rotated with respect to the middle one; such a system can host both flat bands [28,30] and a perfect metal [29].

\section{CONTINUUM MODEL}

We first introduce a continuum model used to calculate the band structure for a monolayer graphene on top of Bernalstacked bilayer graphene [31,32]. An untwisted graphene 
layer has lattice vectors $\mathbf{a}_{1}=a(1,0)$ and $\mathbf{a}_{2}=a(1 / 2, \sqrt{3} / 2)$ with $a=0.246 \mathrm{~nm}$. The reciprocal lattice vectors are $\mathbf{G}_{1}=$ $\frac{2 \pi}{a}(1,-1 / \sqrt{3})$ and $\mathbf{G}_{2}=\frac{2 \pi}{a}(0,2 / \sqrt{3})$. The rotated layers have reciprocal lattice vectors $\mathbf{G}_{\ell, i}=R( \pm \theta / 2) \mathbf{G}_{i}$, where $R( \pm \theta / 2)$ denotes rotation by $\pm \theta / 2$, and we choose a positive twist angle for layer $\ell=1$ and a negative one for layers $\ell=2,3$. The reciprocal Moiré vectors are given by

$$
\mathbf{G}_{i}^{M}=R(-\theta / 2) \mathbf{G}_{i}-R(+\theta / 2) \mathbf{G}_{i} .
$$

The Moiré unit cell lattice vectors are defined by the relation $\mathbf{L}_{i}^{M} \cdot \mathbf{G}_{j}^{M}=2 \pi \delta_{i j}$, where the Moiré lattice constant is $\left|\mathbf{L}_{i}^{M}\right|=$ $\frac{a}{2 \sin \theta / 2}$. The $K$ and $K^{\prime}$ points of the valley $\xi= \pm 1$ are given by $\mathbf{K}_{\ell}^{(\xi)}=-\xi\left(2 \mathbf{G}_{\ell, 1}+\mathbf{G}_{\ell, 2}\right) / 3$.

To describe the low-energy physics of the system, we employ the continuum model that extends the one developed for tBG graphene (see Refs. [7,33,34]). We consider two graphene sheets (layers 1 and 2) that comprise a tBG and add a third, AB-stacked graphene layer aligned with layer 2 [25-27,35]. Retaining the couplings between adjacent layers, we arrive at the following $6 \times 6$ Bloch Hamiltonian matrix for a single valley $\xi$ [in the basis of wavefunction amplitudes on $\mathrm{A}, \mathrm{B}$ sublattices in the three layers $\left.\left(A_{1}, B_{1}, A_{2}, B_{2}, A_{3}, B_{3}\right)\right]$

$$
H^{(\xi)}=\left(\begin{array}{ccc}
H_{1} & U^{\dagger} & 0 \\
U & H_{2} & B^{\dagger} \\
0 & B & H_{3}
\end{array}\right),
$$

where in the continuum approximation the intralayer blocks are

$$
H^{(\xi)}=\left(\begin{array}{cc}
0 & -v \pi^{\dagger} \\
-v \pi & 0
\end{array}\right)
$$

where the $\pi$ terms, given by

$$
\pi=\left[R( \pm \theta / 2)\left(\mathbf{k}-\mathbf{K}_{\ell}^{(\xi)}\right)\right](\xi, i),
$$

depend on the twist angle of the layer, as well as the valley index $\xi$. The matrix $U$ is the effective local interlayer coupling between the twisted layers 1 and 2,

$$
\begin{aligned}
U^{(\xi)}= & \left(\begin{array}{cc}
u & u^{\prime} \\
u^{\prime} & u
\end{array}\right)+\left(\begin{array}{cc}
u & u^{\prime} \omega^{-\xi} \\
u^{\prime} \omega^{\xi} & u
\end{array}\right) e^{i \xi \mathbf{G}_{1}^{M} \cdot \mathbf{r}} \\
& +\left(\begin{array}{cc}
u & u^{\prime} \omega^{\xi} \\
u^{\prime} \omega^{-\xi} & u
\end{array}\right) e^{i \xi\left(\mathbf{G}_{1}^{M}+\mathbf{G}_{2}^{M}\right) \cdot \mathbf{r}} .
\end{aligned}
$$

The matrix $B$ describes the coupling between layers 2 and 3 , and, due to their crystalline alignment, is local in momentum:

$$
B^{(\xi)}=\left(\begin{array}{cc}
-v_{4} \pi & t_{1} \\
-v_{3} \pi^{\dagger} & -v_{4} \pi
\end{array}\right) .
$$

Based on Refs. [7,33,36] for the twisted bilayer parameters and [32] for the AB-stacked bilayer parameters, we set the hoppings $t=-2.61 \mathrm{eV}, t_{1}=0.361 \mathrm{eV}, t_{3}=0.283 \mathrm{meV}$, and $t_{4}=0.140 \mathrm{eV}$. The Dirac velocities are related to the bare hoppings by $v_{i}=t_{i} \sqrt{3} a / 2$. The Moiré hopping is $u^{\prime}=110 \mathrm{meV}$ and $u=0.5 u^{\prime}$. To model the effect of a hBN substrate, we include a $10-\mathrm{meV}$ sublattice symmetry breaking mass term on the first layer [21].

Note that we do not include intervalley scattering, which is in principle symmetry allowed. However, as is the case for $\mathrm{tBG}$, the magnitude of intervalley scattering decreases exponentially with the distance in momentum space between the valleys [7]. Therefore, for small twist angles studied here, the intervalley scattering is several orders of magnitude smaller than other couplings and the bandwidth and can safely be ignored.

\section{FLAT BANDS AND THEIR TOPOLOGY}

The lower symmetry of tMBG compared to the tBG implies that the Dirac cones in the flat bands are no longer symmetry protected. Both at the $\mathbf{K}$ point of the mini-Brillouin zone (mBZ), corresponding to the position of the Dirac cone in the monolayer, as well as at the $\mathbf{K}^{\prime}$ point, corresponding to the position of the band touching in the $\mathrm{AB}$ stacked bilayer, we see a gap opening between the flat bands in a broad range of twist angles.

This situation is markedly different from TBG, where (within the continuum approximation) the interlayer coupling keeps the Dirac cones intact and only renormalizes the Dirac velocity $[7,17]$. Consequently, tMBG does not have a discrete sequence of 'magic angle' where the Dirac velocity vanishes. Nonetheless, we find that tMBG exhibits two flat bands in each valley, separated by a sizable gap from other conduction and valence bands. The flat bands exists in a relatively wide range of angles from $\theta=0.88^{\circ}$ to $\theta=1.5^{\circ}$, where their bandwidth never exceeds $45 \mathrm{meV}$. Furthermore, in the range $1^{\circ}<\theta<1.4^{\circ}$ their bandwidth is smaller than the gaps to other bands, as shown in Fig. 1(a).

The band structure in this regime is illustrated Fig. 1(d). Each valley contains two bands that overlap in a range of energy values. Interestingly, the lower band is topologically trivial whereas the top band has a nonzero Chern number $\mathrm{C}= \pm 1$, with the sign being opposite for the two valleys. The Chern numbers are computed using the method of Ref. [37]. We note that the dispersion of the flat bands is significantly affected by the inclusion of smaller hopping parameters; however, their total Chern number conforms to the general expectations for twisted $N+M$ layer graphene [25].

The distribution of the Berry curvature for the flat bands in the mini-Brillouin zone is shown in Fig. 2. Often, as is the case, e.g., in the Haldane model [38], the Berry curvature is concentrated in the vicinity of gapped Dirac points. Examination of the Berry curvature distribution for both trivial and Chern bands in tMBG indicates the presence of three gapped Dirac points at non-highly-symmetric points in the Brillouin zone. As a result, the Berry curvature is highly inhomogeneous in the BZ.

\section{TUNABILITY OF FLAT BANDS BY GATES}

Next, we investigate the tunability of the flat bands by gates, finding that both their dispersion and topological properties can be efficiently controlled. The gate-induced displacement field is modeled by including a potential $-\delta / 2$ on the first layer and $+\delta / 2$ on the third layer. The resulting band structures for a particular value of twist angle and bias $\delta=$ $\pm 50 \mathrm{meV}$, illustrated in Figs. 1(c) and 1(e), exhibit indirect band gaps between flat bands. Interestingly, this also leads to 

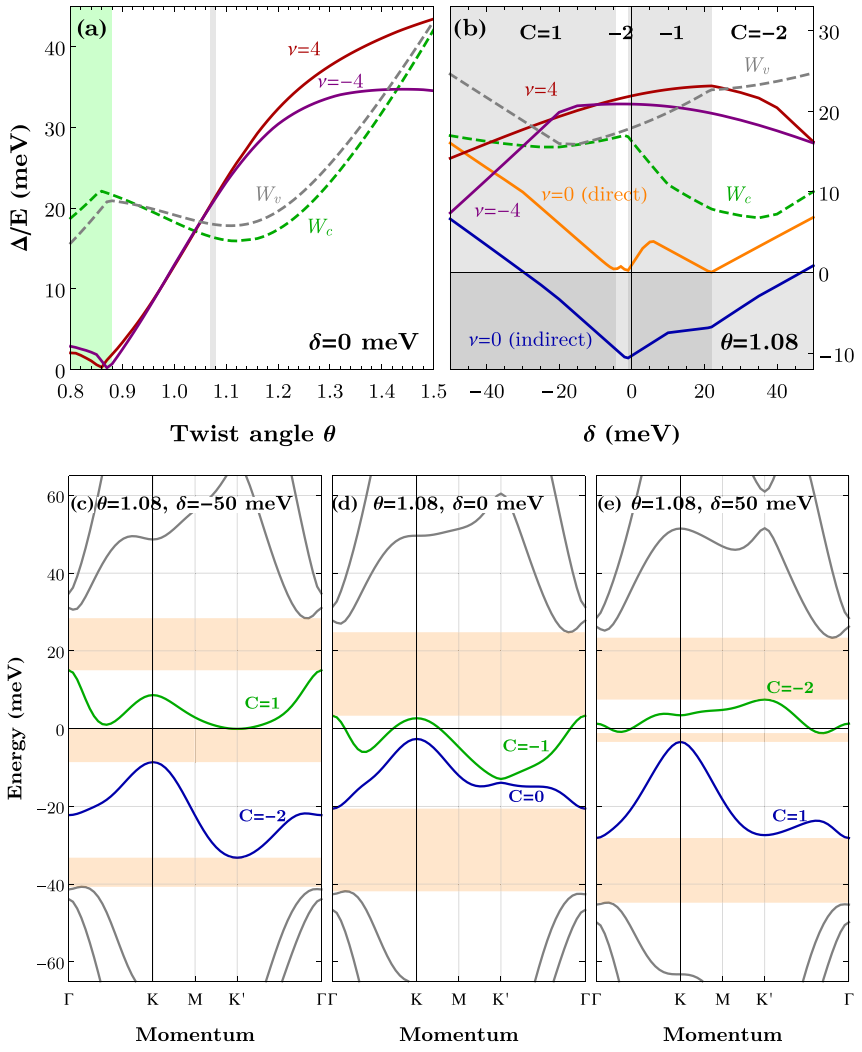

FIG. 1. Twisted monolayer-bilayer graphene is a topologically nontrivial flat band system. (a) The bandwidth of the flat bands (dashed lines, $W_{c}$ for upper flat band and $W_{v}$ for lower flat band) and the superlattice gaps (at fillings $v=-4$ and $v=4$ ) as a function of twist angle. Below $\theta=0.88^{\circ}$, the flat bands are no longer separated from the other bands. (b) The flat bands can be tuned by applying a perpendicular electric field $\delta$, which causes a sequence of topological transitions. The Chern number of the upper flat band in the valley $\xi=1$ goes from $C=1$ at large negative fields to $C=-2$ at large positive fields. We also plot the direct and indirect gap between the two bands (at filling $v=0$ ), revealing that for large fields the two flat bands are well separated without closing the superlattice gaps at $v \pm 4$. [(c)-(e)] The dispersion of the flat bands in one valley $(\xi=1)$ for twist angle $\theta=1.08^{\circ}$ and various displacement field $\delta$. The gaps are indicated by a shaded region. Notice that the total valley Chern number remains constant, consistent with Ref. [25].

a more uniform distribution of the Berry's curvature in the mBZ (Fig. 2).

The gap between conduction and valence bands (both direct and indirect) changes substantially as a function of applied perpendicular displacement field and twist angle $\theta$; see Fig. 1(b). While changing the twist angle does not close the gap, under a change of displacement field the system undergoes several topological transitions. In the regimes where the conduction and valence band also have a positive indirect band gap, the conduction band has either $C=1$ for $D<0$ or $C=-2$ for $D>0$. This is consistent with the calculations of Ref. [36].

\section{RESPONSE TO A MAGNETIC FIELD}

A nonzero Chern number per valley gives rise to a distinct response to a perpendicular magnetic field. According to the

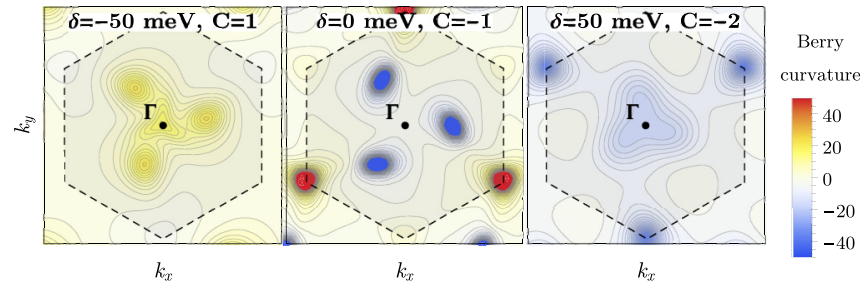

FIG. 2. The Berry curvature in the mini-Brillouin zone for the upper flat band in the $\xi=1$ valley at a twist angle $\theta=1.08^{\circ}$. For $\delta=0 \mathrm{meV}$, there are clear signatures of three gapped Dirac cones away from high-symmetry points, making the Berry curvature highly inhomogeneous throughout the mBZ. In contrast, at large fields $\delta$ the Berry curvature is more uniform and thus more analogous to a Landau level.

Streda formula [39], $\mathrm{C}=\frac{\partial n}{\partial B}$ and therefore the magnetic field reduces (increases) the number of states in a band with a negative (positive) Chern number. For tMBG, this implies that a magnetic field causes valley polarization.

In order to quantify this effect, we computed the spectrum of tMBG in the presence of a magnetic field. Previous works [40-43] have developed complementary approaches to study the behavior of tBG in a magnetic field. We used the method introduced by Ref. [42], which amounts to projecting the interlayer coupling onto a basis set of single-layer Landau level wave functions. The details of our method are outlined in Appendix A.

In Fig. 3, we display the energy spectrum of the system as a function of the magnetic field (the "Hofstadter butterfly" [44]). The right and left halves of the plot describe the evolution of the spectrum in $K$ and $K^{\prime}$ valleys, respectively, in the positive magnetic filed. Flipping the sign of the field interchanges the valleys.

We observe that the states in the flat bands remain well separated from the other bands. It is also clear that a positive field decreases, and a negative field increases the total number

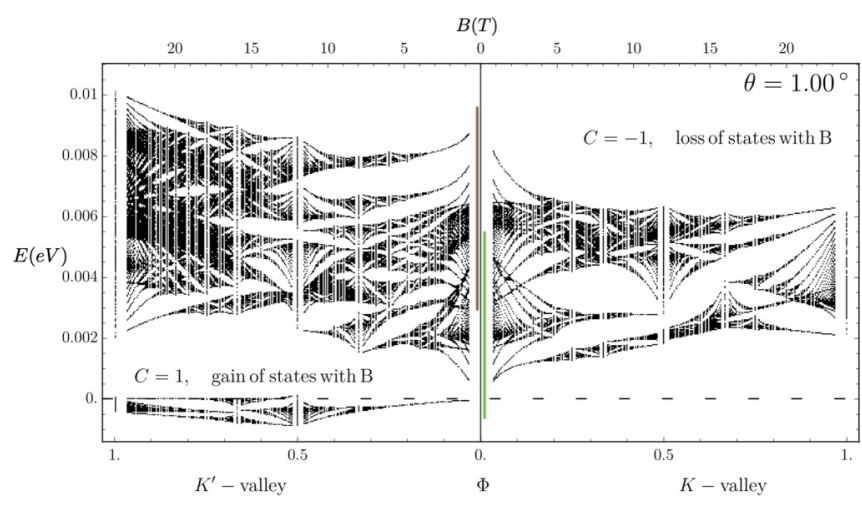

FIG. 3. The energy levels of tMBG in a magnetic field (up to one flux per unit cell, or $B=23.95 \mathrm{~T}$ ), for valley $\xi=1, \theta=1.00^{\circ}$ and only the flat bands, without electric field $(\delta=0 \mathrm{meV})$. An applied positive magnetic field clearly decreases the number of available states, as is expected due to the net Chern number $\mathrm{C}=-1$. The response in the other valley $\xi=-1$ can be obtained by changing the sign of the applied field. 


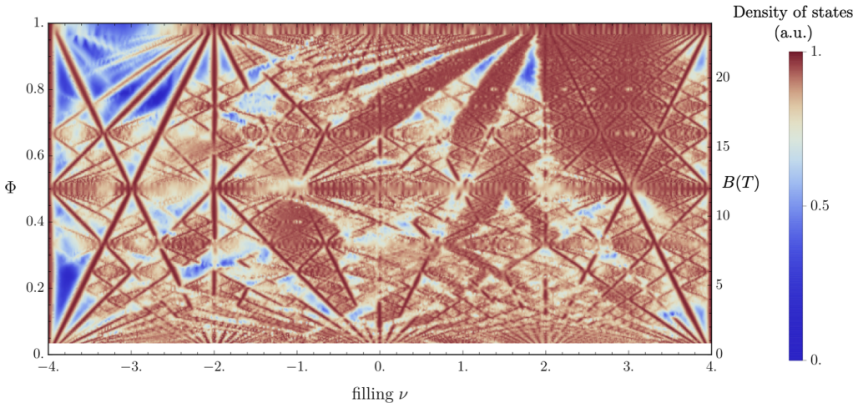

FIG. 4. The density of states as a function of band filling $v$ and magnetic field $B$ in tMBG, also known as a Wannier plot, based on Fig. 3. We show results up to one flux per Moiré unit cell (corresponding to $B=23.95 \mathrm{~T}$, with $\delta=0 \mathrm{meV}$. The filling is measured relative to charge neutrality, so $|v|=4$ means complete filling or emptying of the flat bands.

of states in the valley $C=-1$. This flow of states is compensated by the opposite process in the $C=1$ valley.

We gain additional insight into the field response by converting the Hofstadter butterfly into a Wannier plot [45], which shows the density of states [46] as a function of band filling and applied magnetic field; see Fig. 4. The full range of filling for the flat bands ranges from $v=-4$ to $v=4$, and spin splitting is neglected.

Recall that in a Wannier plot, trivial bands will generate simple Landau "fans" of insulating states around the band edges. The presence of a Dirac cone gives rise to a special sequence of Landau fans with slope $\pm 1, \pm 3, \pm 5, \ldots$.. Bands with nontrivial Chern numbers, on the other hand, lead to an asymmetric behavior due to the valley-field coupling that becomes more pronounced as the field increases. This is clearly seen for $\mathrm{MBG}$, where the density of states is significantly higher close to $v=-4$, washing out the Landau fans originating from other integer fillings. We want to emphasize also the special nature of the inverted Landau fan around $v=2$ and fields close to one flux quantum per Moiré unit cell, where rather than a fan of insulating states a fan of conducting states is observed. This is a common effect in systems where topological and trivial bands occupy the same energy window.

\section{INTERACTION EFFECTS}

The tunable flat bands of tMBG provide a promising playground for interaction-induced states. To identify the range of angles most favorable to observe correlated insulators, we have performed a comparison of the Hartree-Fock energies of variational ground states at several integer filling factors. We used a method similar to previous works on interactioninduced state in tBG [21,22,47-50]. The screened Coulomb interaction $V(r)=\frac{e^{2}}{4 \pi \epsilon_{0} \epsilon r} e^{-r / d}$, with screening length $d=$ $40 \mathrm{~nm}$ and dielectric constant $\epsilon=20$, is projected onto the upper flat bands' wave functions obtained from the continuum model discussed above. A comprehensive account of the formalism and details of the numerical procedure can be found in Appendix B.

We focus on biased samples, with $\delta= \pm 50 \mathrm{meV}$, since in this regime a gap between flat bands develops already at the single-particle level. Moreover, the distribution of Berry's curvature is relatively uniform, suggesting an analogy with Landau levels and the phenomenon of quantum Hall ferromagnetism that favors interaction-induced and spin- and/or valley-polarized states. With this in mind, we exclude spontaneous intervalley coherence or spin mixing in our numerical procedure, meaning the correlation matrix $P^{\left(s s^{\prime}\right)}$ (see Appendix B) is diagonal in band space (because we only consider the upper flat band) and diagonal in spin space. The Hartree term simplifies significantly with this assumption since there is no contribution from intervalley scattering. For both the Fock and Hartree contributions, we introduce a cutoff in $|\mathbf{q}|$ because the strength of $V$ falls rapidly with increasing momentum, and such a cutoff will dramatically speed up calculation. We calculated the ground state for $v=1,2,3$, with dielectric constant $\epsilon=10,20$ for a range of twist angles. The results are shown in Fig. 5.

In agreement with our intuition, these variational calculations indicate that at $v=1$, a fully spin- and valley-polarized state is energetically favored. Such a state is a quantum anomalous Hall (QAH) insulator. For $\delta=50 \mathrm{meV}$, a QAH state at $v=1$ is found to be a ground state for a broad range of twist angles, $\theta \in\left(1^{\circ} ; 1.5^{\circ}\right)$, while for $\delta=-50 \mathrm{meV}$ it is favored in a slightly smaller range, $\theta \in\left(1^{\circ} ; 1.4^{\circ}\right)$. In the former case, the Hall conductivity is predicted to be $\sigma_{x y}= \pm 2 e^{2} / h$, while in the latter case $\pm e^{2} / h$. The positive displacement field regime is expected to be most favorable for the observation of the correlated states, since the bands are flattest while gaps between them are largest. QAH states are also found to be favorable via a similar mechanism at $v=3$.

At $v=2$, we find two possible ground states: either fully valley polarized or fully spin polarized. The fully spinpolarized state would not break time-reversal symmetry and thus would not show a QAH, but the spin-unpolarized state will show a QAH effect. At the variational level, these states are degenerate, and consideration of smaller lattice terms is required to lift this degeneracy.

Note that even though the energy gain is smaller for $\delta>0$, the symmetry-broken states are stable for a longer window when the field is positive. This is consistent with Fig. 1, which shows that the bandwidth of the upper flat band is smaller for positive field.

Recently, Zhu et al. [52] argued that orbital Chern insulators, in contrast to ferromagnetic Chern insulators, should be susceptible to electric switching. Our calculations predict at filling $v=1$ a subtle competition between a spin-polarized state and a spin- and valley-polarized state. For parameters where the spin-polarized state has the lowest energy, namely for strong screening and positive displacement field, we therefore expect a Chern insulator state that is not amenable to electric switching, whereas for other parameters and fillings it should be.

\section{OUTLOOK}

To summarize, we studied the flat bands in a single-layer graphene placed on top of an AB-stacked bilayer with twist. Including smaller interlayer hoppings modifies the dispersion of the bands while maintaining their total nonzero Chern 

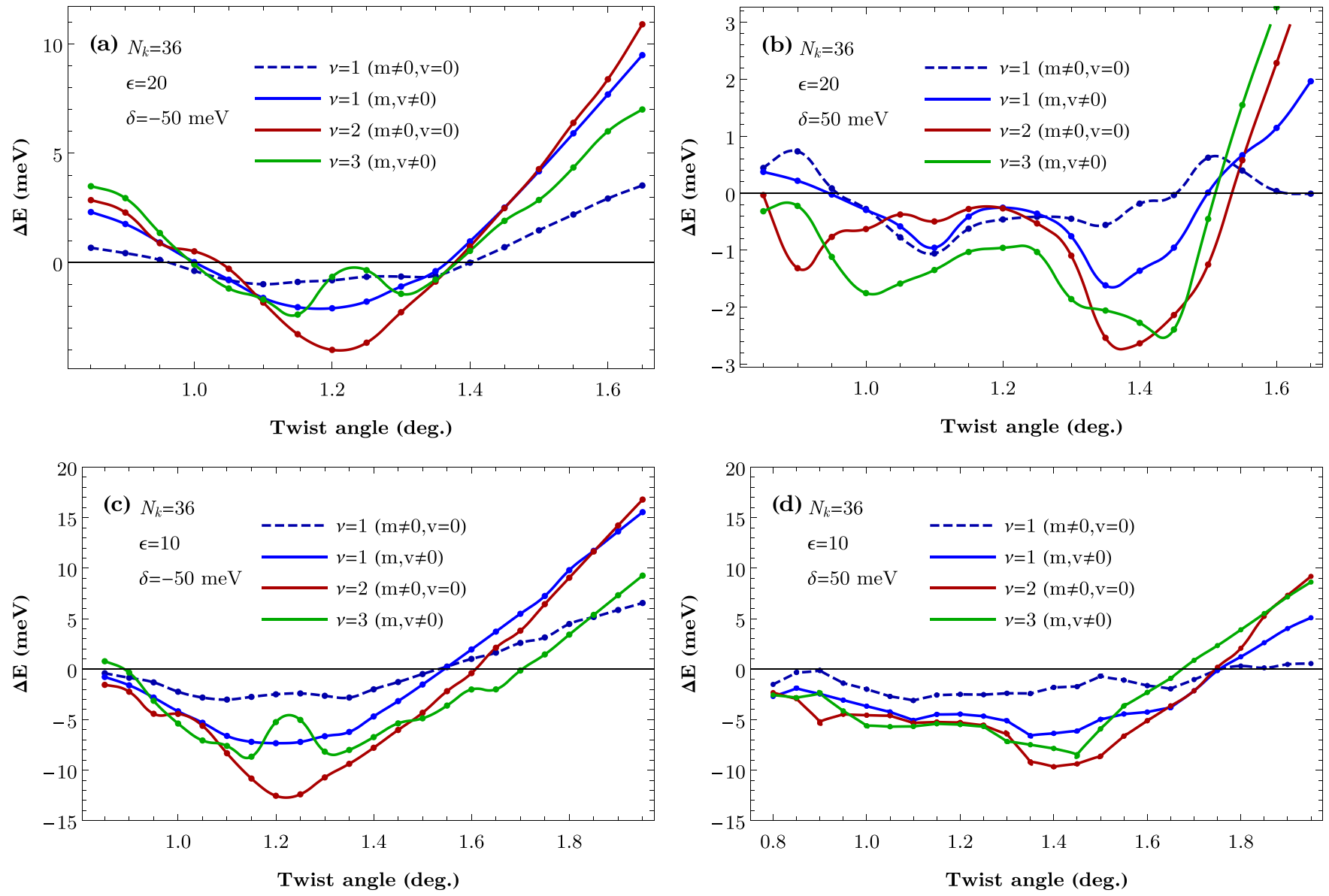

FIG. 5. The mean field energy of the most favorable symmetry broken states at integer fillings, as a function of twist angle. The mean field energy contains both the kinetic energy and the Hartree-Fock interaction energy. The states considered have valley polarization $v$, spin polarization $m$, or both. These results are calculated with $N_{k}=36$ and $\delta= \pm 50 \mathrm{meV}$. For dielectric constant $\epsilon=20$ [panels (a) and (b)], we find that symmetry-broken states are stable in a range of twist angles, 1 to $1.4^{\circ}$ for negative field $\delta=-50 \mathrm{meV}$, and $\theta \lesssim 1.5^{\circ}$ for positive electric field $\delta=50 \mathrm{meV}$. The twist angle range for which symmetry-broken states are observed is consistent with Ref. [51]. Note that for $v=1$, a subtle competition exists between the ferromagnetic state (dashed, $m \neq 0, v=0$ ) and the spin- and valley-polarized state (solid line, $m \neq 0$ and $v \neq 0$ ). Upon decreasing screening to $\epsilon=10$ [panels (c) and (d)], the stability of the correlated phases increases to the point that the critical twist angle is between $1.5^{\circ}$ and $1.7^{\circ}$, depending on the filling.

number, first found within an idealized model of $N+M$ twisted graphene [25]. The topological character of the bands persists for a broad range of twist angles. This, combined with a remarkable tunability of bands by an applied perpendicular field, makes TGBG a versatile platform for realizing controllable, topological bands, and experimentally studying the interplay of interactions and Berry curvature of electronic wave functions.

Using variational Hartree-Fock calculations, we found that interactions favor spin- and/or valley-polarized states at integer filling factors. In particular, the spin- and valley-polarized state at $v=1$ exhibits quantum anomalous Hall effect with a Hall conductivity $\sigma_{x y}= \pm e^{2} / h, \pm 2 e^{2} / h$ that can be tuned by perpendicular electric field. These physics of interactioninduced states in bands with nonzero Chern number carries certain similarity [53] to quantum Hall ferromagnets, and in the future it would be interesting to study the effects of inhomogeneous distribution of Berry's curvature, as well as the energetics of spin and valley textures, such as skyrmions.
Note added. While this paper was being prepared for submission, we learned of three recent experiments $[36,51,54]$ that report the observation of flat bands and signatures of interaction-induced insulating states in tMBG. In particular, Ref. [54] reports the observation of QAH states at $v=1,3$, in the regime of the displacement fields identified above. The reported values of Hall conductivity are consistent with results reported in Fig. 1.

\section{ACKNOWLEDGMENTS}

We thank Amir Yacoby for inspiring discussions. This work was supported by the Swiss National Science Foundation via an Ambizione grant (L.R.) and a regular project (D.A. and I. P.).

\section{APPENDIX A: PERIODIC CONTINUUM MODELS IN MAGNETIC FIELD}

Here we describe the general framework we used to calculate the Hofstadter butterfly of tMBG. Within the continuum 
approximation, the Hamiltonian of tMBG, described in Sec. II, has the following generic form:

$$
H=H_{0}(\mathbf{k})+\sum_{\mathbf{Q}_{i}} T_{\mathbf{Q}_{i}} e^{i \mathbf{Q}_{\mathbf{i}} \mathbf{r}} .
$$

Here, $H_{0}(\mathbf{k})$ is the translationally invariant part (may be a matrix in some sublattice/layer space) and the vectors $\mathbf{Q}_{i}$ $\left[\mathbf{Q}_{i}=0, \pm g_{2}, \pm\left(g_{1}+g_{2}\right)\right.$ in the present case] belong to a lattice generated by some vectors $\overline{\mathbf{A}}_{1}$ and $\overline{\mathbf{A}}_{2}$ (reciprocal moiré vectors in the present case). The full Hamiltonian is thus invariant under real space translations belonging to the lattice generated by $\mathbf{A}_{1,2}$ with

$$
\mathbf{A}_{i} \overline{\mathbf{A}}_{j}=2 \pi \delta_{i j} .
$$

Now we discuss a generic Hamiltonian (A1) in the presence of a commensurate magnetic field $B$ and work out a framework suitable for the numerical computation of the Hofstadter butterfly of the system. We characterize the magnetic field by the flux per unit cell of the A lattice,

$$
\Phi=B \mathcal{A}=\frac{P}{Q} \Phi_{0},
$$

where $\mathcal{A}$ is the area of the real-space unit cell, $P$ and $Q$ are integers, and $\Phi_{0}$ is the (nonsuperconducting) flux quantum. We also introduce the magnetic length $l_{B}, 1 / l_{B}^{2} \equiv B=2 \pi P / Q \mathcal{A}$.

To describe the effect of the magnetic field on the system, it is convenient to introduce the usual set of magnetic creation and annihilation operators

$$
\begin{aligned}
a, a^{+} & =\frac{l_{B}}{\sqrt{2}}\left(\Pi_{x} \pm i \Pi_{y}\right), \\
b, b^{+} & =\frac{1}{l_{B} \sqrt{2}}(X \mp i Y),
\end{aligned}
$$

where

$$
\Pi_{x(y)}=\mathbf{k}_{x(y)}-e A_{x(y)} / c
$$

are the components of the gauge-invariant momentum while $X$ and $Y$ are guiding-center coordinates of the particle in magnetic field. For a free particle (without the periodic perturbations), the operators $a, a^{+}$move the particle between different Landau levels while operators $b$ and $b^{+}$move the particle between different states within the same Landau level.

The Hamiltonian of the system in magnetic field is obtained from Eq. (A1) by the minimal substitution

$$
\mathbf{k}_{x(y)} \longrightarrow \Pi_{x(y)}, \quad \Pi_{x}=\frac{a+a^{+}}{l_{B} \sqrt{2}}, \quad \Pi_{y}=\frac{a-a^{+}}{i l_{B} \sqrt{2}} .
$$

We assume that the matrices $T_{\mathbf{Q}_{i}}$ are momentum independent. Therefore, the minimal substitution affects only the Hamiltonian $H_{0}$ and is straightforward. Of course, for a generic Hamiltonian $H_{0}$ the usual ambiguity arises: One needs to specify the order of the noncommuting operators $\Pi_{x}$ and $\Pi_{y}$ in the minimal substitution. The tMBG Hamiltonian is linear in momentum and free from this problem.

The full Hamiltonian can then be expressed in terms of the $a$ and $b$ operators. The translationally invariant part is a function of the $a$ operators along. In order to get the periodic part one needs to take into account that the particle coordinates are given by

$$
\begin{gathered}
x=X-l_{b}^{2} \Pi_{y}=\frac{l_{B}}{\sqrt{2}}\left(b+b^{+}+i a-i a^{+}\right), \\
y=Y+l_{b}^{2} \Pi_{x}=\frac{l_{B}}{\sqrt{2}}\left(-i b^{+}+i b+a+a^{+}\right) .
\end{gathered}
$$

The initial periodicity of the Hamiltonian (A1) is broken by the magnetic field. However, in a commensurate field with a denominator $Q$, magnetic translations associated to any sublattice of index $Q$ of the original $\mathbf{A}$ lattice remain as a set of commuting symmetries. Specifically, let us denote by $\mathbf{B}_{1,2}$ two (real-space) vectors generating some (arbitrary chosen) sublattice of index $Q$ (i.e., a sublattice with $Q$ times larger unit cell) in the $\mathbf{A}$ lattice. In our numerical studies, we choose $\mathbf{B}_{1}=Q \mathbf{A}_{1}$ and $\mathbf{B}_{2}=\mathbf{A}_{2}$. The corresponding magnetic translation operators $T_{\mathbf{B}_{1(2)}}$ given by

$$
\begin{aligned}
T_{\mathbf{B}_{\alpha}}= & \exp \left[-\frac{\mathbf{B}_{\alpha}^{2}}{4 l_{B}^{2}}\right] \exp \left[-\frac{\mathbf{B}_{\alpha, x}-i \mathbf{B}_{\alpha, y}}{\sqrt{2} l_{B}} b^{+}\right] \\
& \times \exp \left[\frac{\mathbf{B}_{\alpha, x}+i \mathbf{B}_{\alpha, y}}{\sqrt{2} l_{B}} b\right], \\
\alpha= & 1,2,
\end{aligned}
$$

commute with each other and with the Hamiltonian.

In order to exploit the symmetries (A10), we need to construct a basis of eigenfunctions of the operators $T_{\mathbf{B}_{1,2}}$

$$
T_{\mathbf{B}_{1,2}} \psi_{\mathbf{k}}=e^{i \mathbf{B}_{1,2} \mathbf{k}} \psi,
$$

where the quasimomentum $\mathbf{k}$ now specifies the behavior of the wave function under magnetic translations and belongs to the magnetic Brillouin zone.

We note that the magnetic flux per unit cell of the $\mathbf{B}$ lattice is $P \Phi_{0}$. Correspondingly, for every $\mathbf{k}$ there exist $P$ independent states $\psi_{\mathbf{k}}$ for each Landau level. They can be constructed as follows. We chose another lattice $\mathbf{C}$ (generated by some $\left.\mathbf{C}_{1,2}\right)$ such that the $\mathbf{B}$ lattice is a sublattice of $\mathbf{C}$ with index $P$ (so that the unit cell of the $\mathbf{C}$ is $P$ times smaller than that of $\mathbf{B}$ and encircles one flux quantum). In our numerical studies, we choose $\mathbf{C}_{1}=\mathbf{B}_{1} / P$ and $\mathbf{C}_{2}=\mathbf{B}_{1}$. It is then easy to show that for all $\mathbf{p}$ satisfying

$$
\mathbf{p}=\mathbf{k} \bmod \overline{\mathbf{B}}_{1,2}
$$

the eigenfunctions of the magnetic translations $T_{\mathbf{C}_{1,2}}$ [defined in full analogy to Eq. (A10)]

$$
\psi_{\mathbf{p}, n}=N(\mathbf{p}) \sum_{n_{1}, n_{2}=-\infty}^{+\infty} e^{-i \mathbf{p}\left(n_{1} \mathbf{C}_{1}+n_{2} \mathbf{C}_{2}\right)} T_{\mathbf{C}_{1}}^{n_{1}} T_{\mathbf{C}_{2}}^{n_{2}}|\Omega, n\rangle
$$

are also eigenfunctions of $T_{\mathbf{B}_{1,2}}$ corresponding to the quasimomentum k. In Eqs. (A12) and (A13), the vectors $\overline{\mathbf{B}}_{1,2}$ are reciprocal to $\mathbf{B}_{1,2}$, the state $|\Omega, n\rangle \equiv\left[\left(a^{+}\right)^{n} / \sqrt{n !}\right]|\Omega\rangle$ is the vacuum states for $b$ operators in the $n$th Landau level, and $N(\mathbf{p})$ is the normalization factor. It proves convenient to chose the latter according to

$$
N(\mathbf{p})=\left[\frac{\mathbf{C}_{2}^{2}}{2 \mathcal{A}_{\mathbf{C}}}\right]^{1 / 4} z\left[-\frac{\mathbf{C}_{1, x}-i \mathbf{C}_{1, y}}{\mathbf{C}_{2, x}+i \mathbf{C}_{2, y}} \mid-\frac{\mathbf{p C}_{1}}{2 \pi}, \frac{\mathbf{p} \mathbf{C}_{2}}{2 \pi}\right]^{-1},
$$


where $\mathcal{A}_{\mathbf{C}}=\mathcal{A} Q / P$ is the unit cell area for the $\mathbf{C}$ lattice and the function

$$
z\left[\alpha \mid p_{1}, p_{2}\right]=\sum_{r=-\infty}^{\infty} \exp \left[-i \pi \alpha\left(r+p_{2}\right)^{2}+2 \pi i p_{1} r\right]
$$

can be expressed in terms of the Jacobi $\theta$ function.

There are exactly $P$ points in the Brillouin zone of the $\mathbf{C}$ lattice satisfying the condition (A12) and the corresponding $\psi_{\mathbf{p}, n}$ constitute the required basis of eigenfunctions of the magnetic translations $T_{\mathbf{B}_{1,2}}$ within the $n$th Landau level. They are orthogonal for different $\mathbf{p}$ and/or $n$ and with our choice of normalization satisfy

$$
\left\langle\psi_{\mathbf{p}^{\prime}, n^{\prime}} \mid \psi_{\mathbf{p}, n}\right\rangle=\frac{(2 \pi)^{2}}{\mathcal{A}_{\mathbf{C}}} \delta_{n, n^{\prime}} \delta\left(\mathbf{p}-\mathbf{p}^{\prime}\right) .
$$

With the explicit expressions for the basis wave functions (A13) and(A14) at hand, one can reconstruct the the matrix elements of the Hamiltonian (A1) in a magnetic field. The nontrivial part is, of course, the matrix elements of the exponents $e^{i \mathbf{Q r}}$. They can be found with the help of Eqs. (A8) and (A9). The form of the basis wave functions immediately shows that the dependence of the matrix elements on the Landau level indexes and momenta $\mathbf{p}$ factorizes

$$
\left\langle\psi_{n^{\prime} \mathbf{p}^{\prime}}\left|e^{i \mathbf{Q r}}\right| \psi_{n \mathbf{p}}\right\rangle=\frac{(2 \pi)^{2}}{\mathcal{A}_{\mathbf{C}}} \delta_{\overline{\mathbf{C}}}\left(\mathbf{p}^{\prime}-\mathbf{p}-\mathbf{Q}\right) F_{n^{\prime}, n}(\mathbf{Q}) G(\mathbf{p}, \mathbf{Q}),
$$

where the subscript $\overline{\mathbf{C}}$ in the $\delta$ function indicates that its argument should be understood modulo vectors $\overline{\mathbf{C}}_{1,2}$ (reciprocal vectors of the lattice $\mathbf{C}$ ). For the Landau-level dependent part, one finds

$$
\begin{aligned}
& F_{n^{\prime}, n}(\mathbf{Q}) \\
& =e^{-\mathbf{Q}^{2} l_{B}^{2} / 4} \sqrt{n ! n^{\prime} !} \sum_{p=0}^{\min \left(n, n^{\prime}\right)} \frac{\left(q l_{B} / \sqrt{2}\right)^{n^{\prime}-p}\left(-\bar{q} l_{B} / \sqrt{2}\right)^{n-p}}{(n-p) !\left(n^{\prime}-p\right) ! p !}, \\
& q \equiv \mathbf{Q}_{x}+i \mathbf{Q}_{y}, \quad \bar{q} \equiv \mathbf{Q}_{x}-i \mathbf{Q}_{y} .
\end{aligned}
$$

The expression for $G(\mathbf{p}, \mathbf{Q})$ also takes a simple form thanks to our choice of the phase in the normalization (A14)

$$
\begin{aligned}
G(\mathbf{p}, \mathbf{Q})= & \exp \left[\frac{i\left[\mathbf{C}_{1}(\mathbf{Q}+\mathbf{p})\right]\left(\mathbf{C}_{2} \mathbf{p}^{\prime}\right)}{2 \pi}-\frac{i\left(\mathbf{C}_{1} \mathbf{p}\right)\left[\mathbf{C}_{2}(\mathbf{p}+\mathbf{Q})\right]}{2 \pi}\right. \\
& \left.-\frac{i\left(\mathbf{C}_{1} \mathbf{Q}\right)\left(\mathbf{C}_{2} \mathbf{Q}\right)}{4 \pi}\right] .
\end{aligned}
$$

Equations (A17), (A18), and (A19) constitute the main results of this Appendix. They allow a straightforward implementation of numerical analysis of an arbitrary Hamiltonian (A1) in the regime of not too weak magnetic fields where the the Hilbert space of the problem can be truncated in the Landau level index. In particular, this applies to tMBG introduced here. Note the large flexibility of the presented formalism with respect to the possible choice of the lattices $\mathbf{B}$ and $\mathbf{C}$ that can potentially be used to exploit the point-group symmetries (if any) of the Hamiltonian (A1).

\section{APPENDIX B: COULOMB INTERACTIONS IN HARTREE-FOCK}

We will now describe in detail how the Hartree-Fock calculation was performed for the continuum model of tMBG. We follow largely the same methods employed in similar studies of twisted bilayer graphene [21,22,47-50].

\section{Fourier transform in a continuum model}

We first review the definition of the Fourier transforms in a continuum system. Consider a system with area $\Omega$, in particular $N_{\mathrm{UC}}$ copies of the moiré unit cell with area $\Omega_{M}$, so $\Omega=N_{\mathrm{UC}} \Omega_{M}$. The electron field $\psi_{\alpha s}(\mathbf{x})$ is defined at every point in space $\mathbf{x}$ with some flavor index $\alpha s$ (contains spin $s$, layer, and sublattice). We define the Fourier transform on a system with volume $\Omega$ as

$$
\psi_{\alpha s}(\mathbf{x})=\frac{1}{\Omega} \sum_{\mathbf{k}} e^{i \mathbf{k} \cdot \mathbf{x}} \psi_{\mathbf{k} \alpha s}
$$

where the momenta $k$ are of the form $k=\frac{2 \pi}{L} n$ with $n$ being an integer and $L$ being the linear size of the system (so that $\Omega=L^{d}$ for a $d$-dimensional hypercube). The momenta are not bounded, in contrast to a system with sites and a discrete Fourier transform where the number of momenta is equal to the number of sites. In the case of $N_{\mathrm{UC}}$ copies of a moiré unit cell, the possible values of $\mathbf{k}$ will be $\mathbf{k}=\tilde{\mathbf{k}}+\mathbf{G}$ where $\tilde{\mathbf{k}} \in \mathrm{mBZ}$ lives in the mini-Brillouin zone and $\mathbf{G}$ is a moiré reciprocal lattice vector. The number of $\tilde{\mathbf{k}}$ points in the $\mathrm{mBZ}$ $N_{k}$ is equal to the number of moiré unit cell copies in real space,

$$
N_{k}=N_{\mathrm{UC}} .
$$

For clarity, from now on we will refer to a momentum in the mini-BZ with a tilde on it, like $\tilde{\mathbf{k}}$.

The inverse Fourier transform is now

$$
\psi_{\mathbf{k} \alpha s}=\int_{\Omega} d^{2} \mathbf{x} e^{-i \mathbf{k} \cdot \mathbf{x}} \psi_{\alpha s}(\mathbf{x})
$$

\section{Coulomb interaction}

The Coulomb interactions are given by the operator

$$
\hat{V}=\frac{1}{2} \int d^{2} \mathbf{x} d^{2} \mathbf{y} V(\mathbf{x}-\mathbf{y}) \hat{n}(\mathbf{x}) \hat{n}(\mathbf{y}),
$$

where $\hat{n}(\mathbf{x})=\sum_{\alpha s} \psi_{\alpha s}^{\dagger}(\mathbf{x}) \psi_{\alpha s}(\mathbf{x})$ is the coarse-grained electronic density summed over both sublattice $\alpha$ and spin $s$. For $V(r)$, we take the screened Coulomb interaction, $V(r)=$ $\frac{e^{2}}{4 \pi \epsilon r} e^{-r / d}$. The Fourier transform of the screened Coulomb interaction is

$$
\begin{aligned}
V(k) & =\int_{\Omega} d^{2} \mathbf{x} e^{-i \mathbf{k} \cdot \mathbf{x}} V(|\mathbf{x}|) \\
& =\int_{\Omega} r d r d \theta e^{-i k r \cos \theta} \frac{e^{2}}{4 \pi \epsilon r} e^{-r / d} \\
& =\frac{e^{2}}{2 \epsilon} \int d r J_{0}(k r) e^{-r / d} \\
& =\frac{e^{2}}{2 \epsilon} \frac{1}{\sqrt{k^{2}+d^{-2}}} .
\end{aligned}
$$


We set a screening length of $d=40 \mathrm{~nm}$ and the dielectric constant $\epsilon$ will be varied.

The Coulomb operator now reads explicitly in terms of the momentum-based electron operators $\psi_{\mathbf{k} \alpha s}$ :

$$
\begin{aligned}
\hat{V} & =\frac{1}{2} \int d^{2} \mathbf{r} \frac{1}{\Omega^{3}} \sum_{\mathbf{k} \mathbf{k}^{\prime} \mathbf{q} \alpha s \alpha^{\prime} s^{\prime}} \psi_{\mathbf{k}+\mathbf{q} \alpha s}^{\dagger} \psi_{\mathbf{k} \alpha s} \psi_{\mathbf{k}^{\prime}-\mathbf{q} \alpha^{\prime} s^{\prime}}^{\dagger} \psi_{\mathbf{k}^{\prime} \alpha^{\prime} s^{\prime}} V(r) e^{-i \mathbf{q r} \mathbf{r}} \\
& =\frac{1}{2 \Omega} \sum_{\mathbf{q}} V(q) \rho_{\mathbf{q}} \rho_{-\mathbf{q}},
\end{aligned}
$$

where the momentum-space particle density is defined as

$$
\rho_{\mathbf{q}}=\frac{1}{\Omega} \sum_{\mathbf{k} \alpha s} \psi_{\mathbf{k}+\mathbf{q} \alpha s}^{\dagger} \psi_{\mathbf{k} \alpha s}
$$

\section{Bloch functions}

The Bloch Hamiltonian for a given momentum $\tilde{\mathbf{k}}$ inside the mini-Brillouin zone $(\mathrm{mBZ})$ is a $6 N_{\mathrm{BZ}} \times 6 N_{\mathrm{BZ}}$ matrix, where $N_{\mathrm{BZ}}$ is the number of mini-BZ copies (each with a momentum associated with $\tilde{\mathbf{k}}+\mathbf{G} \equiv \tilde{\mathbf{k}}+n_{1} \mathbf{G}_{1}^{M}+n_{2} \mathbf{G}_{2}^{M}$ ). An eigenstate of the Bloch Hamiltonian is thus a vector $\left\{\phi_{n ; \mathbf{G} \alpha}^{(\tilde{\mathbf{k}})}\right\}$, where the eigenvector is labeled by the band index $n$ and the momentum $\tilde{\mathbf{k}}$, and the vector has $6 N_{\mathrm{BZ}}$ components, labeled by the miniBrillouin zone index $\mathbf{G}$ and the sublattice index $\alpha=\ell \sigma=1 \mathrm{~A}$, $1 \mathrm{~B}, 2 \mathrm{~A}$, etc.
The operator to create a state with band index $n$ at $\mathrm{mBZ}$ momentum $\tilde{\mathbf{k}}$ and $\operatorname{spin} s$ is

$$
c_{\tilde{\mathbf{k}} n}^{\dagger}=\sum_{\mathbf{G} \alpha} \phi_{n ; \mathbf{G} \alpha}^{(\tilde{\mathbf{k}})} \psi_{\tilde{\mathbf{k}}+\mathbf{G}, \alpha s}^{\dagger} .
$$

Since there are as many bands (labeled $n$ ) as reciprocal vectors $\mathbf{G}$ times sublattices $\alpha$, the eigenvectors $\phi_{n ; \mathbf{G} \alpha}^{(\tilde{\mathbf{k}})}$ together form a unitary matrix. This means

$$
\begin{gathered}
\sum_{n}\left(\phi_{n ; \mathbf{G}^{\prime} \alpha^{\prime}}^{(\tilde{\mathbf{k}})}\right)^{*} \phi_{n ; \mathbf{G} \alpha}^{(\tilde{\mathbf{k}})}=\delta_{\mathbf{G}^{\prime} \alpha^{\prime}=\mathbf{G} \alpha}, \\
\sum_{\mathbf{G} \alpha}\left(\phi_{n ; \mathbf{G} \alpha}^{(\tilde{\mathbf{k}})}\right)^{*} \phi_{n^{\prime} ; \mathbf{G} \alpha}^{(\tilde{\mathbf{k}})}=\delta_{n n^{\prime}} .
\end{gathered}
$$

These identities can be useful to invert expressions Eq. (B12). We find that the operators $\psi_{\mathbf{k} \alpha s}^{\dagger}$ can be expressed in terms of the operators for the bands $n$,

$$
\psi_{\mathbf{k}, \alpha s}^{\dagger}=\sum_{n}\left(\phi_{n ; \mathbf{G}_{k} \alpha}^{(\tilde{\mathbf{k}})}\right)^{*} c_{\tilde{\mathbf{k}} n s}^{\dagger},
$$

where we implicitly defined the projection of $\mathbf{k}$ into the $\mathrm{mBZ}$ as $\tilde{\mathbf{k}}$, and $\mathbf{G}_{k}$ as the corresponding reciprocal lattice vector.

\section{Hartree-Fock decoupling}

The Coulomb energy contains sums over momenta $\mathbf{k}, \mathbf{k}^{\prime}$, and $\mathbf{q}$, which all can be decoupled in a sum over a momentum in the $\mathrm{mBZ}$ and a reciprocal lattice vector,

$$
\frac{1}{\Omega} \sum_{\mathbf{k}} \rightarrow \frac{1}{N_{k} \Omega_{M}} \sum_{\tilde{\mathbf{k}} \mathbf{G}_{k}} .
$$

So let us continue with Eq. (B10) and write now the Coulomb energy operator in terms of the $c$ operators:

$$
\begin{aligned}
& \hat{V}=\frac{1}{2 \Omega^{3}} \sum_{\tilde{\mathbf{q}} \mathbf{G}_{q}} V\left(\left|\mathbf{q}+\mathbf{G}_{q}\right|\right)\left(\sum_{\tilde{\mathbf{k}} \mathbf{G}_{k} \alpha s} \psi_{\tilde{\mathbf{k}}+\tilde{\mathbf{q}}+\mathbf{G}_{k}+\mathbf{G}_{q} \alpha s}^{\dagger} \psi_{\tilde{\mathbf{k}}+\mathbf{G}_{k} \alpha s}\right)\left(\sum_{\tilde{\mathbf{k}^{\prime}} \mathbf{G}_{k^{\prime}} \alpha^{\prime} s^{\prime}} \psi_{\tilde{\mathbf{k}^{\prime}}-\tilde{\mathbf{q}}+\mathbf{G}_{k}-\mathbf{G}_{q} \alpha^{\prime} s^{\prime}}^{\dagger} \psi_{\tilde{\mathbf{k}}^{\prime}+\mathbf{G}_{k^{\prime}} \alpha^{\prime} s^{\prime}}\right) \\
& =\frac{1}{2 \Omega^{3}} \sum_{\tilde{\mathbf{q}} \mathbf{G}_{q} \tilde{\mathbf{k}} \mathbf{G}_{k} \alpha s \tilde{\mathbf{k}}^{\prime} \mathbf{G}_{k^{\prime}} \alpha^{\prime} s^{\prime}} V\left(\left|\mathbf{q}+\mathbf{G}_{q}\right|\right) \sum_{n_{1} n_{2} n_{3} n_{4}} c_{\tilde{\mathbf{k}}+\tilde{\mathbf{q}} n_{1} s}^{\dagger} c_{\tilde{\mathbf{k}} n_{2} s} c_{\tilde{\mathbf{k}^{\prime}}-\tilde{\mathbf{q}} n_{3} s^{\prime}}^{\dagger} c_{\tilde{\mathbf{k}}^{\prime} n_{4} s^{\prime}}\left(\phi_{n_{1} ; \mathbf{G}_{k}+\mathbf{G}_{q} \alpha}^{(\tilde{\mathbf{k}}+\tilde{\mathbf{q}})}\right)^{*}\left(\phi_{n_{2} ; \mathbf{G}_{k} \alpha}^{(\tilde{\mathbf{k}})}\right)\left(\phi_{n_{3} ; \mathbf{G}_{k^{\prime}}-\mathbf{G}_{q} \alpha^{\prime}}^{\left(\tilde{\mathbf{k}^{\prime}}\right) \tilde{\mathbf{q}^{\prime}}}\right)^{*}\left(\phi_{n_{4} ; \mathbf{G}_{k^{\prime}} \alpha^{\prime}}^{\left(\tilde{\mathbf{k}^{\prime}}\right)}\right. \\
& =\frac{1}{2 \Omega} \sum_{\mathbf{q} \tilde{\mathbf{k}} \tilde{\mathbf{k}}^{\prime} s s^{\prime}} \sum_{n_{1} n_{2} n_{3} n_{4}} c_{\tilde{\mathbf{k}}+\tilde{\mathbf{q}} n_{1} s}^{\dagger} c_{\tilde{\mathbf{k}} n_{2} s} c_{\tilde{\mathbf{k}^{\prime}}-\tilde{\mathbf{q}} n_{3} s^{\prime}}^{\dagger} c_{\tilde{\mathbf{k}}^{\prime} n_{4} s^{\prime}} \Lambda_{\mathbf{q} ; n_{1} n_{2}}(\tilde{\mathbf{k}}) \Lambda_{-\mathbf{q} ; n_{3} n_{4}}\left(\tilde{\mathbf{k}}^{\prime}\right),
\end{aligned}
$$

where we defined the form factor matrix following [22] as

$$
\Lambda_{\mathbf{q} ; n_{1} n_{2}}(\tilde{\mathbf{k}}) \equiv \frac{1}{\Omega} \sum_{\mathbf{G}_{k} \alpha}\left(\phi_{n_{1} ; \mathbf{G}_{k}+\mathbf{G}_{q} \alpha}^{(\tilde{\mathbf{k}}+\tilde{\mathbf{q}})}\right)^{*}\left(\phi_{n_{2} ; \mathbf{G}_{k} \alpha}^{(\tilde{\mathbf{k}})}\right)
$$

which is defined for any momentum $\mathbf{q}$ (not restricted to the mBZ). Notice that $\Lambda_{-\mathbf{q}}(\tilde{\mathbf{k}}+\tilde{\mathbf{q}})=\left[\Lambda_{\mathbf{q}}(\tilde{\mathbf{k}})\right]^{\dagger}$.

The Hartree contribution to the energy is calculated as follows. We define the correlation matrix

$$
P_{n_{1} ; n_{2}}^{\left(s s^{\prime}\right)}(\tilde{\mathbf{k}})=\left\langle c_{\tilde{\mathbf{k}} n_{1} s}^{\dagger} c_{\tilde{\mathbf{k}} n_{2} s^{\prime}}\right\rangle
$$

and we assume no translational symmetry breaking. Then the Hartree term becomes

$$
E_{H}=\frac{1}{2 \Omega} \sum_{\mathbf{G}_{q} \tilde{\mathbf{k}} \tilde{\mathbf{k}}^{\prime} s s^{\prime}} V\left(\left|\mathbf{G}_{q}\right|\right) \operatorname{Tr}\left[\Lambda_{\mathbf{G}_{q}}(\tilde{\mathbf{k}}) P^{(s s)}(\tilde{\mathbf{k}})\right] \operatorname{Tr}\left[\Lambda_{\mathbf{G}_{q}}^{\dagger}\left(\tilde{\mathbf{k}}^{\prime}\right) P^{\left(s^{\prime} s^{\prime}\right)}\left(\tilde{\mathbf{k}}^{\prime}\right)\right]
$$


Similarly, the Fock term is expressed as

$$
E_{F}=-\frac{1}{2 \Omega} \sum_{\mathbf{q} \tilde{\mathbf{k}} s s^{\prime}} V(|\mathbf{q}|) \operatorname{Tr}\left[P^{\left(s s^{\prime}\right)}(\tilde{\mathbf{k}}+\tilde{\mathbf{q}}) \Lambda_{\mathbf{q}}^{*}(\tilde{\mathbf{k}}) P^{\left(s^{\prime} s\right)}(\tilde{\mathbf{k}}) \Lambda_{\mathbf{q}}^{\top}(\tilde{\mathbf{k}})\right] .
$$

Note that these are the same equations as (S26) of Ref. [22].

[1] Y. Cao, V. Fatemi, S. Fang, K. Watanabe, T. Taniguchi, E. Kaxiras, and P. Jarillo-Herrero, Nature (London) 556, 43 (2018).

[2] Y. Cao, V. Fatemi, A. Demir, S. Fang, S. L. Tomarken, J. Y. Luo, J. D. Sanchez-Yamagishi, K. Watanabe, T. Taniguchi, E. Kaxiras, R. C. Ashoori, and P. Jarillo-Herrero, Nature (London) 556, 80 (2018).

[3] Y. Cao, D. Chowdhury, D. Rodan-Legrain, O. Rubies-Bigordà, K. Watanabe, T. Taniguchi, T. Senthil, and P. Jarillo-Herrero, Phys. Rev. Lett. 124, 076801 (2020).

[4] A. H. MacDonald, Physics 12, 12 (2019).

[5] A. Kerelsky, L. J. McGilly, D. M. Kennes, L. Xian, M. Yankowitz, S. Chen, K. Watanabe, T. Taniguchi, J. Hone, C. Dean, A. Rubio, and A. N. Pasupathy, Nature (London) 572, 95 (2019).

[6] M. Yankowitz, S. Chen, H. Polshyn, Y. Zhang, K. Watanabe, T. Taniguchi, D. Graf, A. F. Young, and C. R. Dean, Science 363, 1059 (2019).

[7] R. Bistritzer and A. H. MacDonald, Proc. Natl. Acad. Sci. U.S.A. 108, 12233 (2011).

[8] Y. Cao, D. Rodan-Legrain, O. Rubies-Bigordà, J. M. Park, K. Watanabe, T. Taniguchi, and P. Jarillo-Herrero, arXiv:1903.08596v1.

[9] G. Chen, A. L. Sharpe, E. J. Fox, Y.-H. Zhang, S. Wang, L. Jiang, B. Lyu, H. Li, K. Watanabe, T. Taniguchi, Z. Shi, T. Senthil, D. Goldhaber-Gordon, Y. Zhang, and F. Wang, Nature 579, 56 (2020).

[10] G. Chen, A. L. Sharpe, P. Gallagher, I. T. Rosen, E. Fox, L. Jiang, B. Lyu, H. Li, K. Watanabe, T. Taniguchi, J. Jung, Z. Shi, D. Goldhaber-Gordon, Y. Zhang, and F. Wang, Nature (London) 572, 215 (2019).

[11] B. L. Chittari, G. Chen, Y. Zhang, F. Wang, and J. Jung, Phys. Rev. Lett. 122, 016401 (2019).

[12] L. Wang, E.-M. Shih, A. Ghiotto, L. Xian, D. A. Rhodes, C. Tan, M. Claassen, D. M. Kennes, Y. Bai, B. Kim, K. Watanabe, T. Taniguchi, X. Zhu, J. Hone, A. Rubio, A. Pasupathy, and C. R. Dean, arXiv:1910.12147v1.

[13] J. C. W. Song, P. Samutpraphoot, and L. S. Levitov, Proc. Natl. Acad. Sci. U.S.A. 112, 10879 (2015).

[14] Y.-H. Zhang, D. Mao, Y. Cao, P. Jarillo-Herrero, and T. Senthil, Phys. Rev. B 99, 075127 (2019).

[15] H. C. Po, H. Watanabe, and A. Vishwanath, Phys. Rev. Lett. 121, 126402 (2018).

[16] H. C. Po, L. Zou, T. Senthil, and A. Vishwanath, Phys. Rev. B 99, 195455 (2019).

[17] Z. Song, Z. Wang, W. Shi, G. Li, C. Fang, and B. A. Bernevig, Phys. Rev. Lett. 123, 036401 (2019).

[18] J. Ahn, S. Park, and B.-J. Yang, Phys. Rev. X 9, 021013 (2019).

[19] A. L. Sharpe, E. J. Fox, A. W. Barnard, J. Finney, K. Watanabe, T. Taniguchi, M. A. Kastner, and D. GoldhaberGordon, Science 365, 605 (2019).
[20] M. Serlin, C. L. Tschirhart, H. Polshyn, Y. Zhang, J. Zhu, K. Watanabe, T. Taniguchi, L. Balents, and A. F. Young, Science 367, 900 (2020).

[21] N. Bultinck, S. Chatterjee, and M. P. Zaletel, Phys. Rev. Lett. 124, 166601 (2020).

[22] N. Bultinck, E. Khalaf, S. Liu, S. Chatterjee, A. Vishwanath, and M. P. Zaletel, arXiv:1911.02045.

[23] Y.-H. Zhang, D. Mao, and T. Senthil, Phys. Rev. Res. 1, 033126 (2019).

[24] C. Repellin, Z. Dong, Y. H. Zhang, and T. Senthil, Phys. Rev. Lett. 124, 187601 (2020).

[25] J. Liu, Z. Ma, J. Gao, and X. Dai, Phys. Rev. X 9, 031021 (2019).

[26] Z. Ma, S. Li, Y.-W. Zheng, M.-M. Xiao, H. Jiang, J.-H. Gao, and X. C. Xie, arXiv:1905.00622.

[27] Y. Park, B. Lingam Chittari, and J. Jung, Phys. Rev. B 102, 035411 (2020).

[28] E. Khalaf, A. J. Kruchkov, G. Tarnopolsky, and A. Vishwanath, Phys. Rev. B 100, 085109 (2019).

[29] C. Mora, N. Regnault, and B. A. Bernevig, Phys. Rev. Lett. 123, 026402 (2019).

[30] X. Li, F. Wu, and A. H. MacDonald, arXiv:1907.12338 [condmat.mtrl-sci].

[31] E. McCann and M. Koshino, Rep. Prog. Phys. 76, 056503 (2012).

[32] J. Jung and A. H. MacDonald, Phys. Rev. B 89, 035405 (2014).

[33] M. Koshino, N. F. Q. Yuan, T. Koretsune, M. Ochi, K. Kuroki, and L. Fu, Phys. Rev. X 8, 031087 (2018).

[34] L. Balents, SciPost Phys. 7, 048 (2019).

[35] E. Suárez Morell, M. Pacheco, L. Chico, and L. Brey, Phys. Rev. B 87, 125414 (2013).

[36] S. Chen, M. He, Y.-H. Zhang, V. Hsieh, Z. Fei, K. Watanabe, T. Taniguchi, D. H. Cobden, X. Xu, C. R. Dean, and M. Yankowitz, arXiv:2004.11340v1.

[37] T. Fukui, Y. Hatsugai, and H. Suzuki, J. Phys. Soc. Jpn. 74, 1674 (2005).

[38] F. D. M. Haldane, Phys. Rev. Lett. 61, 2015 (1988).

[39] P. Streda, J. Phys. C: Solid State Phys. 15, L1299 (2000).

[40] R. Bistritzer and A. H. MacDonald, Phys. Rev. B 84, 035440 (2011).

[41] B. Lian, F. Xie, and B. A. Bernevig, arXiv:1811.11786v2.

[42] K. Hejazi, C. Liu, and L. Balents, Phys. Rev. B 100, 035115 (2019).

[43] Y.-H. Zhang, H. C. Po, and T. Senthil, Phys. Rev. B 100, 125104 (2019).

[44] D. R. Hofstadter, Phys. Rev. B 14, 2239 (1976).

[45] F. H. Claro and G. H. Wannier, Phys. Rev. B 19, 6068 (1979).

[46] In this work, limiting ourself to qualitative analysis, we compute the density of states by phenomenologically ascribing to the states in the Landau levels a finite lifetime (energy and flux independent). This procedure allows to highlight the insulating 
states on the $B-v$ phase diagram. Microscopic analysis of the elastic scattering processes in realistic samples is needed to make our predictions quantitative.

[47] M. Xie and A. H. MacDonald, arXiv:1812.04213v1.

[48] J. Liu and X. Dai, arXiv:1911.03760v1.

[49] T. Cea and F. Guinea, Phys. Rev. B 102, 045107 (2020).

[50] T. Cea, N. R. Waler, and F. Guinea, arXiv:1906.10570v1.

[51] Y. Shi, S. Xu, M. M. Al Ezzi, N. Balakrishnan, A. GarciaRuiz, B. Tsim, C. Mullan, J. Barrier, N. Xin, B. A. Piot,
T. Taniguchi, K. Watanabe, A. Carvalho, A. Mishchenko, A. K. Geim, V. I. Fal'ko, S. Adam, A. H. C. Neto, and K. S. Novoselov, arXiv:2004.12414v1.

[52] J. Zhu, J.-J. Su, and A. H. MacDonald, arXiv:2001.05084.

[53] A. Kumar, R. Roy, and S. L. Sondhi, Phys. Rev. B 90, 245106 (2014).

[54] H. Polshyn, J. Zhu, M. A. Kumar, Y. Zhang, F. Yang, C. L. Tschirhart, M. Serlin, K. Watanabe, T. Taniguchi, A. H. MacDonald, and A. F. Young, arXiv:2004.11353. 